\title{
Complex Gaussian quadrature for oscillatory integral transforms
}

\author{
Andreas Asheim and Daan Huybrechs
}

Report TW 594, May 2011

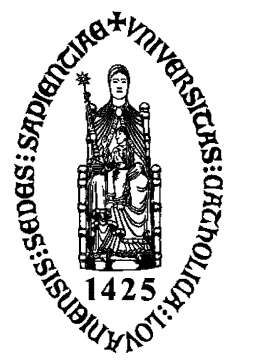

\section{Katholieke Universiteit Leuven}

Department of Computer Science

Celestijnenlaan 200A - B-3001 Heverlee (Belgium) 


\title{
Complex Gaussian quadrature for oscillatory integral transforms
}

\author{
Andreas Asheim and Daan Huybrechs \\ Report TW 594, May 2011 \\ Department of Computer Science, K.U.Leuven
}

\begin{abstract}
The classical theory of Gaussian quadrature assumes a positive weight function. We will show that in some cases Gaussian rules can be constructed with respect to an oscillatory weight, yielding methods with complex quadrature nodes and positive weights. These rules are well suited for highly oscillatory integrals because they attain optimal asymptotic order. We show that for the Fourier oscillator this approach yields the numerical method of steepest descent, a method with optimal asymptotic order that has previously been proposed for this class of integrals. However, the approach readily extends to more general kernels, such as Bessel functions that appear as the kernel of the Hankel transform.
\end{abstract}

Keywords : integral transforms, orthogonal polynomials, numerical integration AMS(MOS) Classification : Primary : 65R10, Secondary : 30E20, 41A60, $42 \mathrm{C} 05$. 


\title{
Complex Gaussian quadrature for oscillatory integral transforms
}

\author{
Andreas Asheim $\dagger$, Daan Huybrechs $\ddagger$ \\ $\dagger, \ddagger$ Departement Computerwetenschappen, K.U. Leuven, Belgium \\ Email: Andreas.Asheim@cs.kuleuven.be
}

May, 2011

\begin{abstract}
The classical theory of Gaussian quadrature assumes a positive weight function. We will show that in some cases Gaussian rules can be constructed with respect to an oscillatory weight, yielding methods with complex quadrature nodes and positive weights. These rules are well suited for highly oscillatory integrals because they attain optimal asymptotic order. We show that for the Fourier oscillator this approach yields the numerical method of steepest descent, a method with optimal asymptotic order that has previously been proposed for this class of integrals. However, the approach readily extends to more general kernels, such as Bessel functions that appear as the kernel of the Hankel transform.
\end{abstract}

\section{Introduction}

For positive weight functions $h(x)$, it is well known that integrals of the form

$$
\int_{a}^{b} f(x) h(x) \mathrm{d} x
$$

can be approximated efficiently by Gaussian quadrature rules for smooth functions $f(x)$. A positive weight function $h(x)$ implies that, provided moments are finite, such rules exist, and that the quadrature nodes, i.e. the roots of the orthogonal polynomials w.r.t. $h(x)$, lie in the interior of the interval $[a, b]$. Furthermore, the corresponding quadrature weights will be positive, which implies that the quadrature rule is stable.

For integral transforms like the Hankel transform

$$
\left(\mathcal{H}_{\nu} f\right)(\omega):=\int_{0}^{\infty} f(x) J_{\nu}(\omega x) \mathrm{d} x
$$

we are not so blessed. On the same note we could mention transforms with other Bessel functions as kernels, like Hankel functions of the first or second kind, Airy functions, and also Fourier oscillators. The Hankel transform shows up in different applications, primarily by the way of Fourier analysis, since the 
Hankel transform is essentially the 2D Fourier transform of a radial symmetric function. Integrals of functions involving Hankel functions appear in boundary element method formulations of scattering problems[12,3]. Integrals involving Airy functions are encountered in quantum physics under the name of Airy averaging $[6]$.

Wong, in a paper in Numerische Mathematik[17], suggested rewriting Hankel transforms and related transforms as linear combinations of integrals of Hankel functions, with paths of integration deformed to the imaginary axis. This yields two integrals with a positive weight function related to $K_{\nu}$, the modified Bessel function of the second kind. Applying the corresponding Gaussian quadrature rules gives a method that achieves excellent accuracy for analytic $f$. Computation of such rules is discussed in [7]. In this paper we shall, by building on recent developments in the field of highly oscillatory quadrature, re-examine this work. In particular, we shall show that Wong's approach, which involves constructing Gaussian rules for two integrals, can be replaced by a Gaussian rule for the transform itself, and that such rules will be more accurate for large arguments. Moreover, we avoid a difficulty with the above mentioned rewriting of the transform, namely that for $\nu \geq 1$ it involves rewriting $J_{\nu}$ in terms of non-integrable functions. On the other hand, by admitting oscillatory weights, and thereby abandoning much of the theory of Gaussian quadrature, we are moving into unknown territory; we can no longer guarantee existence of rules, nor quadrature nodes in the interior of the interval or positive weights. Nevertheless it should be pointed out that the notion of quadrature nodes contained in the interior of the interval has already been abandoned in Wong's approach since the paths of integration are deformed.

We must stress that these methods require $f$ to be analytic in large parts of the complex plane. Without additional changes, this is likely to make them unsuited for many practical applications where functions with singularities are the most important objects of studies. However, the same problem arises when applying complex Gaussian quadrature in the case $h(x)=e^{i x}$ or $h(x)=e^{i g(x)}$, a case that has received considerable attention in recent years (see for example[11, 1, 14, 13]). In that setting, the stringent analycity requirements can be completely avoided by judiciously adding extra quadrature points on the real line, whilst maintaining the high asymptotic order of accuracy of the underlying Gaussian rules[10]. The extension of this idea to the current setting may lead to efficient, practical and robust evaluation methods of general oscillatory integral transforms. It is this promising prospect that motivates the necessary first step towards that goal: a study of the Gaussian quadrature rules and their associated orthogonal polynomials.

\subsection{Gaussian quadrature}

A quadrature rule with nodes and weights $\left(w_{j}, x_{j}\right), j=1, \ldots, n$ is called Gaussian if it has degree of exactness $2 n-1$, meaning the rule is exact for polynomials up to degree $2 n-1$. Since the nodes of such rules are the zeros of orthogonal polynomials, the existence of such rules depends critically on the existence of orthogonal polynomials. The following theorem is the classical existence and 
uniqueness result for orthogonal polynomials. It can be found in [8].

Theorem 1. Let $h(x)$ be a given weight function. If

$$
(u, v)_{h}=\int u(x) v(x) h(x) \mathrm{d} x
$$

is a positive definite inner product on $\mathbb{P}$, the space of real polynomials, then there exists a unique sequence $\pi_{k}$ of monic orthogonal polynomials w.r.t. $h(x)$.

We only outline the proof here: Define $\pi_{0}=1$, and denote the sequence of monomials $e_{k}(t)=t^{k}, k=0,1, \ldots$ Then by applying Gram-Schmidt's othogonalization procedure, we get the polynomials by the recurrence

$$
\pi_{k}=e_{k}-\sum_{l=0}^{k-1} c_{l} \pi_{l},
$$

where $c_{l}=\frac{\left(e_{k}, \pi_{l}\right)_{h}}{\left(\pi_{l}, \pi_{l}\right)_{h}}$. Now the result follows since the inner product is positive definite, implying existence and uniqueness for the coefficients $c_{l}$.

If $h$ is a positive weight, the pairing $(\cdot, \cdot)_{h}$ is indeed a positive definite inner product, implying existence and uniqueness of the quadrature rule[8, Theorem 1.45]. The condition is sufficient, but not necessary. From the proof of Theorem 1 we can devise a simple corollary, which enables us to talk about orthogonal polynomials for non-positive weights:

Corollary 1. Assume that $\pi_{l}$ is given and $\left(\pi_{l}, \pi_{l}\right)_{h} \neq 0$ for $l=0, \ldots, k-1$. Then $\pi_{k}$ generated by (3) is orthogonal to all $\pi_{l}$ for $l=0, \ldots, k-1$.

From now we view the pairing $(\cdot, \cdot)_{h}$ as a more general bilinear form, thus it is not necessarily an inner product. Still, if the orthogonalisation process terminates we have orthogonal polynomials.

\subsection{Oscillatory quadrature and integral transforms}

Highly oscillatory quadrature methods based on Gaussian quadrature, i.e. numerical steepest descent methods[11], have proved successful for obtaining accurate approximations with high asymptotic order. As an example of this method, consider a complex analytic function $f$ with appropriate decay at infinity. A half-space Fourier integral can be rewritten as

$$
\int_{0}^{\infty} f(x) e^{i \omega x} \mathrm{~d} x=\frac{i}{\omega} \int_{0}^{\infty} f(i p / \omega) e^{-p} \mathrm{~d} p .
$$

This corresponds to deforming the path of integration into the complex plane along the path of steepest descent, which in this case is the imaginary axis. Applying Gauss-Laguerre quadrature on the semi-infinite integral on the right yields a method for which $n$ evaluations of $f$ in the complex plane yields an error with asymptotic order $\mathcal{O}\left(\omega^{-2 n-1}\right)[4]$. Note that the quadrature nodes of this method are located in the complex plane on the path of steepest descent, and they are scaled towards the endpoint 0 for increasing $\omega$. 
A complication arises when one considers an integral of the form

$$
\int_{0}^{\infty} f(x) \sin (\omega x) \mathrm{d} x
$$

since $\sin (x)$ is exponentially large away from the real line, so the method of steepest descent will fail. A natural way to proceed with this integral is to use the identity $\sin (x)=\left(e^{i x}-e^{-i x}\right) / 2 i$ and write it as the sum of two Fourier integrals. However, two issues spring up: First, we have to use twice as many function evaluations as in the purely exponential case if we wish to achieve the same asymptotic order. Secondly, such a splitting is ad-hoc; it requires us to identify a linear combination of functions that decay in the complex plane. When proceeding to more difficult kernels, the way to achieve this might not be obvious.

We show in $\S 3.2$ that it is indeed possible to construct a rule with $\sin (x)$ as a weight. Here we show the equivalence between this sine-rule and another rule with a well defined positive weight function along a path in the complex plane. It is therefore clear that the rule has positive weights and complex quadrature nodes. Thus we refer to such rules as complex Gaussian.

For computing the rule we abandon the notion of positive weight functions: we construct polynomials orthogonal to $\sin (x)$ and locate the zeros of these. This approach can easily be extended to more general kernels, e.g. the kernel of the Hankel transform $J_{\nu}$. A general theory describing the cases for which this procedure succeeds is lacking, and would probably be rather involved. Yet, we identify several cases that can be related to existing theory and several other cases for which the zeros show interesting patterns related to inherent features of the weight function. The asymptotic properties of the resulting rules are similar to that of the (rotated) Gauss-Laguerre applied to the Fourier integral, i.e. the error behaves like $\mathcal{O}\left(\omega^{-2 n-1}\right)$. This is a general property for complex Gaussian rules which we shall prove in section $\S 1.4$.

\subsection{Regularization of moments}

The moments for e.g. the weight $e^{i x}$ over $[0, \infty)$ clearly do not exist in a classical sense. However, we can apply an integral analogue of Abel's limit theorem for power series, and thus assign finite values to the moments. The following lemma is found in [17], and is proved in [16]:

Lemma 1. If the integral $\int_{0}^{\infty} \phi(x) \mathrm{d} x$ converges as an improper Riemann integral, then

$$
\lim _{s \rightarrow 0} \int_{0}^{\infty} \phi(x) e^{-s x} \mathrm{~d} x=\int_{0}^{\infty} \phi(x) \mathrm{d} x .
$$

Now we can specify what we imply by exactness for polynomials.

Definition 1. We say that a quadrature rule $Q[f] \approx \int_{0}^{\infty} f(x) h(x) \mathrm{d} x$ is exact for polynomials up to degree $m$ if

$$
Q\left[x^{j}\right]=\lim _{s \rightarrow 0} \int_{0}^{\infty} x^{j} h(x) e^{-s x} \mathrm{~d} x, \quad j=0, \ldots, m .
$$




\subsection{Asymptotic order of Gaussian rules}

The primary reason for using complex Gaussian rules for evaluating oscillatory integrals is that they attain high asymptotic order. The Lemmas in this section are generalisations of Lemmas 1 and 2 in [4] to allow for more general, nonmonotone $h$.

For the proof we need to recall a generalisation of Watson's Lemma due to Handelsman and Bleistein[2]. We here include a more restrictive version of the theorem, in which we have omitted potential logarithmic singularities of $f$. For the full theorem as well as proofs, see the referred paper.

Theorem 2. Let $I(\omega)$ be defined by

$$
I(\omega)=\int_{0}^{\infty} f(x) h(\omega x) \mathrm{d} x,
$$

where $f$ and $g$ are both $C^{\infty}$ functions on $[0, \infty)$, and assume $f$ has an asymptotic expansion of the form $f(x) \sim x^{\mu} \sum_{k=0}^{\infty} f_{k} x^{k}$ as $x \rightarrow 0$. Further assume one of the following cases,

i) $h(x)$ is exponentially decaying at infinity: $h(x)=\mathcal{O}\left(e^{-c x^{\nu}}\right)$ as $x \rightarrow \infty$, for $c, \nu>0$,

ii) $h(x)$ is an oscillatory kernel at infinity, i.e.

$$
h(x) \sim \exp \left(i \sum_{l=0}^{l<\nu / \delta} b_{l} x^{\nu-\delta l}\right) \sum_{k=0}^{\infty} c_{k} x^{-k}, \quad x \rightarrow \infty,
$$

$\nu>0, \delta>0, b_{l}$ real and $f$ is at worst algebraic at $\infty$ and non-oscillatory.

Then

$$
I(\omega) \sim \omega^{-\mu} \sum_{k=0}^{\infty} f_{k} \omega^{-k-1} M_{k}[h],
$$

where $M_{k}[h]$ are functionals not depending on $\omega$.

Note that the restrictions $i$ ) and $i i$ ) on $h$ are rather mild. All kernels we shall consider in the following will satisfy one of them. Now, the crucial information we need from this theorem is that integrals of the form (5) decay with $\omega$ in much the same way as a Laplace integral, also for oscillatory kernels, with the terms in the asymptotic expansions depending only on the derivatives of $f$ at 0 . From this, the asymptotics of the quadrature error follows.

Lemma 2. Assume a quadrature rule with nodes $\left\{w_{j}, x_{j}\right\}, j=1, \ldots, n$ such that,

$$
\int_{0}^{\infty} x^{m} h(x) \mathrm{d} x=\sum_{j=1}^{n} w_{j} x_{j}^{m}, \quad \text { for } \quad m=0, \ldots, N-1 .
$$

Also assume $h(x)$ satisfies either property $i)$ or ii) in Theorem 2. Then, for $f$ analytic at 0 and $C^{\infty}$ on $[0, \infty)$,

$$
\int_{0}^{\infty} f(x) h(\omega x) \mathrm{d} x-\frac{1}{\omega} \sum_{j=1}^{n} w_{j} f\left(\frac{x_{j}}{\omega}\right)=\mathcal{O}\left(\omega^{-N-1}\right), \quad \omega \rightarrow \infty .
$$


Proof. By the change of variable $t=\omega x$, we get,

$$
\int_{0}^{\infty} x^{m} h(\omega x) \mathrm{d} x=\frac{1}{\omega} \int_{0}^{\infty}(t / \omega)^{m} h(t) \mathrm{d} t=\frac{1}{\omega} \sum_{j=1}^{n} w_{j}\left(\frac{x_{j}}{\omega}\right)^{m}, \quad \text { for } \quad m=0, \ldots, N-1 .
$$

Since $f$ is analytic at zero, it has a convergent Taylor series for $x<R$ with $R>0$,

$$
f(x)=\sum_{l=0}^{\infty} f_{l} x^{l}, \quad x<R .
$$

Denote by $f_{N}(x)$ the series trucated after $N$ terms, $f_{N}(x):=\sum_{l=0}^{N-1} f_{l} x^{l}$, and $f_{e}(x):=f(x)-f_{N}(x)$.

$$
\begin{aligned}
\int_{0}^{\infty} f(x) h(\omega x) \mathrm{d} x-\frac{1}{\omega} \sum_{j=1}^{n} w_{j} f\left(\frac{x_{j}}{\omega}\right)= & \int_{0}^{\infty} f_{N}(x) h(\omega x) \mathrm{d} x-\frac{1}{\omega} \sum_{j=1}^{n} w_{j} f_{N}\left(\frac{x_{j}}{\omega}\right) \\
& +\int_{0}^{\infty} f_{e}(x) h(\omega x) \mathrm{d} x-\frac{1}{\omega} \sum_{j=1}^{n} w_{j} f_{e}\left(\frac{x_{j}}{\omega}\right) \\
= & \int_{0}^{\infty} f_{e}(x) h(\omega x) \mathrm{d} x-\frac{1}{\omega} \sum_{j=1}^{n} w_{j} f_{e}\left(\frac{x_{j}}{\omega}\right),
\end{aligned}
$$

where the last equality follows from the fact the rule is exact for monomials up to degree $N$. From the definition of $f_{e}$ it's clear that $f_{e}(x) \sim x^{N} \sum_{l=0}^{\infty} f_{l+N} x^{l}$ as $x \rightarrow 0$. Therefore, by Theorem 2 ,

$$
\int_{0}^{\infty} f_{e}(x) h(\omega x) \mathrm{d} x=\mathcal{O}\left(\omega^{-N-1}\right)
$$

It remains to prove that the sum is also of the same asymptotic size. This follows by the observation that for $x_{j} / \omega<R$,

$$
f_{e}\left(x_{j} / \omega\right)=f\left(x_{j} / \omega\right)-f_{N}\left(x_{j} / \omega\right)=\sum_{l=N}^{\infty} f_{l} \cdot(x / \omega)^{l}=\mathcal{O}\left(\omega^{-N}\right) .
$$

Adapting this proof on minor points will lead to a proof of the analogue Lemma for integrals over all reals:

Lemma 3. Assume a quadrature rule with nodes $\left\{w_{j}, x_{j}\right\}, j=1, \ldots, n$ such that,

$$
\int_{-\infty}^{\infty} x^{m} h(x) \mathrm{d} x=\sum_{j=1}^{n} w_{j} x_{j}^{m}, \quad \text { for } \quad m=0, \ldots, N-1 .
$$

Then, for $f$ analytic at 0 ,

$$
\int_{-\infty}^{\infty} f(x) h(\omega x) \mathrm{d} x-\frac{1}{\omega} \sum_{j=1}^{n} w_{j} f\left(\frac{x_{j}}{\omega}\right)=\mathcal{O}\left(\omega^{-N-1}\right), \quad \omega \rightarrow \infty
$$

Remark 1. These Lemmas do not necessarily hold for compact intervals. Nevertheless, by rewriting as a difference between two integrals over semi-infinte intervals the lemma can formally be applied. 


\section{Earlier work}

We recall the main results of [17]. By using the identity

$$
J_{\nu}(z)=\frac{1}{2}\left(H_{\nu}^{(1)}(z)+H_{\nu}^{(2)}(z)\right)
$$

the Hankel transform (2) can be rewritten as

$$
\left(\mathcal{H}_{\nu} f\right)(\omega)=\frac{1}{2} \int_{0}^{\infty} f(x) H_{\nu}^{(1)}(z) \mathrm{d} z+\frac{1}{2} \int_{0}^{\infty} f(x) H_{\nu}^{(2)}(z) \mathrm{d} z .
$$

Wong uses this as a basis of an approach for evaluating the Hankel transform. By further using that,

$$
H_{\nu}^{(1)}(z)=\frac{2}{\pi i} e^{-i \pi \nu / 2} K_{\nu}(-i t), \quad H_{\nu}^{(2)}(z)=-\frac{2}{\pi i} e^{i \pi \nu / 2} K_{\nu}(i t),
$$

it is argued that by deforming the integration contours, onto the positive and negative imaginary axis respectively, both integrals can be expressed with a positive weight function, the modified Bessel function $K_{\nu}$, on $[0, \infty)$. Therefore Gaussian rules for integrals of the form

$$
\int_{0}^{\infty} f(x) x^{\mu} K_{\nu}(x) \mathrm{d} x
$$

are suggested. Here $\mu \pm \nu>-1$ is required for moments to exist,

$$
\int_{0}^{\infty} x^{m+\mu} K_{\nu}(x) \mathrm{d} x=2^{m+\mu-1} \Gamma\left(\frac{1}{2}+\frac{m+\mu+\nu}{2}\right) \Gamma\left(\frac{1}{2}+\frac{m+\mu-\nu}{2}\right) .
$$

Thus orthogonal polynomials can then be constructed. Further, by comparing the moments of the Hankel transform,

$$
\int_{0}^{\infty} x^{m} J_{\nu}(\omega x) \mathrm{d} x=2^{m} \frac{\Gamma\left(\frac{1}{2}+\frac{\nu+m}{2}\right)}{\Gamma\left(\frac{1}{2}+\frac{\nu-m}{2}\right) \omega^{m+1}},
$$

with (7), it can be seen that for a combined rule for the Hankel transform the condition $\mu \pm \nu>-1$ can be relaxed to $\mu+\nu>-1$.

Furthermore, Wong proves that,

$$
\int_{0}^{\infty} x^{\mu} f(x) H_{\nu}^{(1)}(\omega x) \mathrm{d} x=\frac{2}{\pi} \frac{e^{i(\mu-\nu) \pi / 2}}{\omega^{\mu+1}} \sum_{k=1}^{n} w_{k} f\left(i t_{k} / \omega\right)+\delta_{n},
$$

where $\left\{w_{j}, x_{j}\right\}_{j=1}^{n}$ are the weights and nodes of the Gaussian quadrature with weight $K_{\nu}$. The error term $\delta_{n}$ has the form

$$
\delta_{n}=C_{n} \omega^{-2 n-\mu-1} f^{(2 n)}(i \xi / \omega), \quad 0<\xi<\infty,
$$

which incorporates the asymptotic behaviour of the rule. For $\mu=0$, this observation agrees with the result of Lemma 2 applied to each of the two integrals in (6), but note that $2 n$ points are thus needed in total to get the same asymptotic order when applying this method to the Hankel transform. 


\section{Complex Gaussian quadrature}

In this section we will present some examples of complex Gaussian rules for some oscillatory kernels. First, in $§ 3.1$, we discuss the connection between the numerical method of steepest descent and complex Gaussian rules for $e^{i x^{r}}$. Further on we discuss rules with the kernel $\sin (x)$ in $\S 3.2, J_{0}(x)$ in $\S 3.3, J_{\nu}(x)$ in $\S 3.4, \operatorname{Ai}(x)$ in $\S 3.5$, and at last some more illustrations in $\S 3.6$. It will be demonstrated that most, but not all, of these rules can be rewritten in terms of rules with positive weight functions along paths in the complex plane.

For the computation of the rules we utilise only evaluation of moments, and then compute orthogonal polynomials by Gram-Schmidt's procedure as explained in $\S 1.1$. Roots are computed with general purpose root finding algorithms for the purposes of our illustration, though we note that in principle they can be found more efficiently as the eigenvalues of the Jacobi matrix constructed from the recurrence coefficients of the orthogonal polynomials[9].

\subsection{Complex Gauss quadrature and numerical steepest descent}

For integrals of the form

$$
\int_{a}^{b} f(x) e^{i \omega g(x)} \mathrm{d} x
$$

the method of steepest descent leads to the evaluation of a set of integrals, one for each asymptotically contributing point, of the form

$$
\int_{-\infty}^{\infty} f(\gamma(t)) \gamma^{\prime}(t) e^{-\omega t^{r}} \mathrm{~d} t
$$

where $\gamma(t)$ is a parametrization of the path of steepest descent. Applying Gaussian quadrature to these integrals is what is often referred to as the numerical method of steepest descent[11]. Now note that if the integral has only one contributing point, we are left with only one such integral. If furthermore $\gamma$ is affine, then the numerical steepest descent method will be exact for $f$ being a polynomial of degree $\leq 2 n-1$ with $n$ function evaluations. This is in other words a Gaussian rule, which is essentially the following result.

In the case of Fourier-like oscillators $e^{ \pm i x^{r}}$ we can compute the moments explicitly,

$$
\lim _{s \rightarrow 0} \int_{0}^{\infty} x^{m} e^{ \pm i x^{r}} e^{-s x} \mathrm{~d} x=\frac{1}{r} e^{\frac{ \pm i(m+1) \pi}{2 r}} \Gamma\left(\frac{m+1}{r}\right) .
$$

Theorem 3. Let $\left\{x_{j}^{r}, w_{j}^{r}\right\}, j=1, \ldots, n$, be the nodes and weights of the $n$-point Gaussian quadrature w.r.t. the weight function $e^{-x^{r}}, r>0$ on the interval $[0, \infty)$. A Gaussian rule w.r.t. the weight function $h(x)=e^{ \pm i x^{r}}$ exists with nodes $\tilde{x}_{j}^{r}=x_{j}^{r} e^{ \pm i \pi /(2 r)}$ and weights $\tilde{w}_{j}^{r}=e^{ \pm i \pi /(2 r)} w_{j}^{r}$.

Proof. The proof goes by examining the moments. First we note that the moments corresponding to the weight $e^{-x^{r}}$ are,

$$
\mu_{m}^{r}:=\int_{0}^{\infty} x^{m} e^{-x^{r}} \mathrm{~d} x=\frac{1}{r} \Gamma\left(\frac{m+1}{r}\right) .
$$


If we let $\tilde{\mu}_{m}^{r}$ denote the moments for the weight $e^{ \pm i x^{r}}$ we then see that

$$
\tilde{\mu}_{m}^{r}=e^{\frac{ \pm i(m+1) \pi}{2 r}} \mu_{m}^{r} .
$$

Requiring exactness for monomials gives

$$
\tilde{\mu}_{m}^{r}=\sum_{j=1}^{n}\left(\tilde{x}_{j}^{r}\right)^{m} \tilde{w}_{j}^{r}=e^{\frac{ \pm i(m+1) \pi}{2 r}} \sum_{j=1}^{n}\left(x_{j}^{r}\right)^{m} w_{j}^{r}, \quad m=0, \ldots, 2 n-1 .
$$

By inspection it is confirmed that $\tilde{x}_{j}^{r}=x_{j}^{r} e^{ \pm i \pi /(2 r)}$ and $\tilde{w}_{j}^{r}=e^{ \pm i \pi /(2 r)} w_{j}^{r}$ are solutions to these equations.

The theorem essentially illustrates that the limit in the left hand side of (8) is equivalent to regularizing the integral by deforming the path onto the steepest descent path in the complex plane.

For the case of corresponding integrals over the whole real line we only state the result. The proof is analogous to that of Theorem 3

Theorem 4. Let $\left\{x_{j}^{r}, w_{j}^{r}\right\}, j=1, \ldots, n$, be the nodes and weights of the $n$-point Gaussian quadrature w.r.t. the weight function $e^{-x^{r}}$ for even $r>0$ on the interval $(-\infty, \infty)$. The Gaussian rule w.r.t. the weight function $h(x)=e^{ \pm i x^{r}}$ exists with nodes $\tilde{x}_{j}^{r}=x_{j}^{r} e^{ \pm i \pi /(2 r)}$ and weights $\tilde{w}_{j}^{r}=w_{j}^{r} e^{ \pm i \pi /(2 r)}$.

Remark 2. For the case of odd $r$, the situation is quite different, since the path of steepest descent is no longer smooth. Rules for these cases have been examined in [5].

\subsection{The sine rule}

A Gaussian rule with $\sin (x)$ as weight is interesting in being a case where the Gaussian approach and numerical steepest descent yield different rules. The moments can be computed,

$$
\lim _{s \rightarrow 0} \int_{0}^{\infty} x^{m} \sin (x) e^{-s x} \mathrm{~d} x= \begin{cases}(-1)^{m / 2} \Gamma(m+1), & m \text { even } \\ 0, & m \text { odd }\end{cases}
$$

Theorem 5. Let $\left\{x_{j}, w_{j}\right\}_{j=1}^{n}$ be a Gaussian w.r.t. the weight function $\frac{1}{2} e^{-\sqrt{t}} t^{-\frac{1}{2}}$ on $[0, \infty)$. The rule $\left\{\tilde{x}_{j}, \tilde{w}_{j}\right\}_{j=1}^{2 n}$, where $\left\{\tilde{x}_{j}\right\}_{j=1}^{2 n}=\left\{i \sqrt{x_{j}}\right\} \cup\left\{-i \sqrt{x_{j}}\right\}_{j=1}^{n}$ and $\left\{\tilde{w}_{j}\right\}_{j=1}^{2 n}=\left\{\frac{1}{2} w_{j}\right\} \cup\left\{\frac{1}{2} w_{j}\right\}_{j=1}^{n}$, is Gaussian w.r.t. the weight $\sin (x)$ on $[0, \infty)$.

Proof. First consider

$$
\int_{0}^{\infty} f(x) \sin (x) \mathrm{d} x=\frac{1}{2 i} \int_{0}^{\infty} f(x) e^{i x} \mathrm{~d} x-\frac{1}{2 i} \int_{0}^{\infty} f(x) e^{-i x} \mathrm{~d} x .
$$

For $f$ being analytic in the right half-plane and suitably decaying at infinity we can deform paths of integration onto the positive and negative imaginary axis respectively for the two integrals, giving

$$
\begin{aligned}
\int_{0}^{\infty} f(x) \sin (x) \mathrm{d} x & =\frac{1}{2} \int_{0}^{\infty}(f(i t)+f(-i t)) e^{-t} \mathrm{~d} t \\
& =\int_{0}^{\infty} f_{E}(i t) e^{-t} \mathrm{~d} t
\end{aligned}
$$


where $f_{E}(x)=(f(x)+f(-x)) / 2$ is the even part of $f(x)$.

Next, the rule $\left\{x_{j}, w_{j}\right\}_{j=1}^{n}$ has the property,

$$
\sum_{j=1}^{n} w_{j} x_{j}^{m}=\frac{1}{2} \int_{0}^{\infty} t^{m} e^{-\sqrt{t}} t^{-1 / 2} \mathrm{~d} t=\int_{0}^{\infty} x^{2 m} e^{-x} \mathrm{~d} x, \quad m=0, \ldots, 2 n-1,
$$

which implies that it is a Gaussian rule for the integral (10) in the sense that it exactly integrates even polynomials up to order $4 n-2$ with $n$ function evaluations. Now, applying the rule to (10) gives the evaluation points $\left\{i \sqrt{x_{j}}\right\}$ with corresponding weights $\left\{\frac{1}{2} w_{j}\right\}$, plus $\left\{-i \sqrt{x_{j}}\right\}$ with corresponding weights $\left\{\frac{1}{2} w_{j}\right\}$. This rule is exact for all polynomials up to order $4 n-1$ with $2 n$ function evaluations, since the even part of a polynomial of degree $4 n-1$ is of degree $4 n-2$.

Note that the moments for the weight $\frac{1}{2} e^{-\sqrt{t}} t^{-\frac{1}{2}}$ are also easily computed,

$$
\frac{1}{2} \int_{0}^{\infty} e^{-\sqrt{t}} t^{-\frac{1}{2}} t^{m} \mathrm{~d} t=\Gamma(2 m+1),
$$

and comparing moments will give the same result. Also note that since this weight function is positive we know that the nodes for this rule are contained in $[0, \infty)$, and that the weights are positive. By the theorem this translates to the sine-rule having purely imaginary nodes and positive weights.

Figure 1a) shows 25 points of the sine rule. An alternative way of evaluating the same transform is by taking the decomposition (9) and applying the two rotated Laguerre quadratures described in $\S 3.1$. Taking $n$ points for each will yield the same asymptotic properties as $n$ points from the sine rule. Figure 1a) shows the layout of these points for $n=25$.

(a)

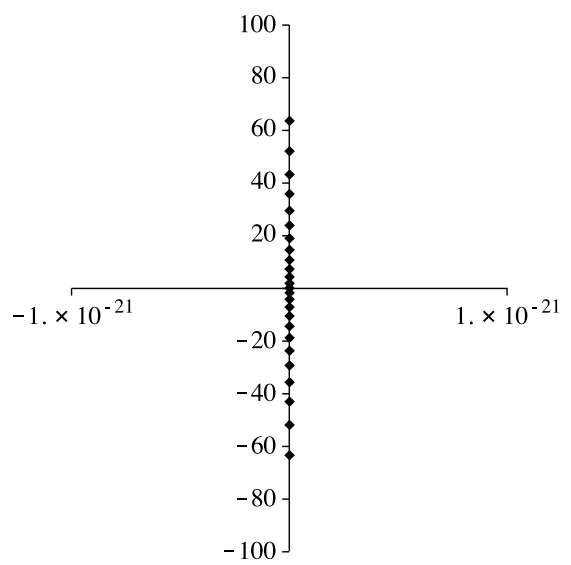

Figure 1: (a) 25 points of the sine-rule. (b) 25 points of $e^{i x}$-rule and 25 points of $e^{-i x}$-rule.

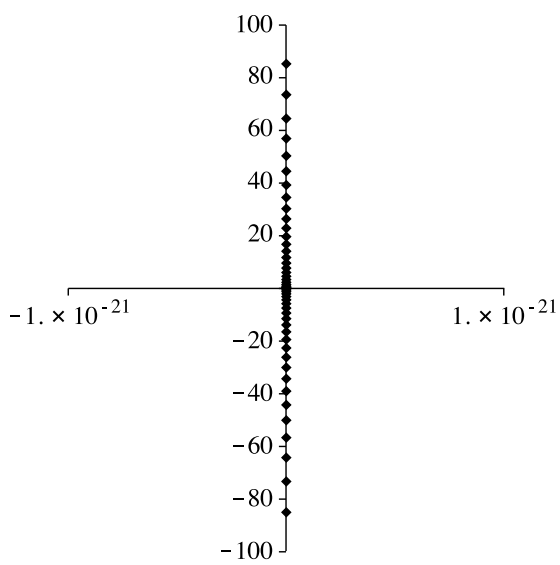

(b) 


\subsection{The $J_{0}$ rule}

In this case of the Hankel transform of order zero the moments can also be obtained explicitly,

$$
\lim _{s \rightarrow 0} \int_{0}^{\infty} x^{m} J_{0}(x) e^{-s x} \mathrm{~d} x=2^{m} \frac{\Gamma\left(\frac{1}{2}+\frac{m}{2}\right)}{\Gamma\left(\frac{1}{2}-\frac{m}{2}\right)} .
$$

A correspondence between the complex rule obtained from this and a Gaussian rule with a positive weight function can also be identified in this case.

Theorem 6. Let $\left\{x_{j}, w_{j}\right\}_{j=1}^{n}$ be a Gaussian rule w.r.t. the weight function $\frac{1}{2} K_{0}(\sqrt{t}) t^{-\frac{1}{2}}$ on $[0, \infty)$. The rule $\left\{\tilde{x}_{j}, \tilde{w}_{j}\right\}_{j=1}^{2 n}$, where $\left\{\tilde{x}_{j}\right\}_{j=1}^{2 n}=\left\{i \sqrt{x_{j}}\right\} \cup$ $\left\{-i \sqrt{x_{j}}\right\}_{j=1}^{n}$ and $\left\{\tilde{w}_{j}\right\}_{j=1}^{2 n}=\left\{\frac{1}{2} w_{j}\right\} \cup\left\{\frac{1}{2} w_{j}\right\}_{j=1}^{n}$, is Gaussian w.r.t. the weight $J_{0}(x)$ on $[0, \infty)$.

Proof. By the identity

$$
J_{0}(z)=\frac{1}{i \pi}\left(K_{0}(-i z)-K_{0}(i z)\right), \quad \operatorname{Arg}(z) \leq \pi / 2,
$$

we get

$$
\int_{0}^{\infty} f(x) J_{0}(x) \mathrm{d} x=\frac{1}{i \pi}\left(\int_{0}^{\infty} f(x) K_{0}(-i x) \mathrm{d} x-\int_{0}^{\infty} f(x) K_{0}(i x) \mathrm{d} x\right) .
$$

With $f$ being analytic in the right half-plane and suitably decaying at infinity we can deform paths of integration onto the positive and negative imaginary axis respectively for the two integrals, giving

$$
\begin{aligned}
\int_{0}^{\infty} f(x) J_{0}(x) \mathrm{d} x & =\frac{1}{\pi} \int_{0}^{\infty}(f(i t)+f(-i t)) K_{0}(t) \mathrm{d} x \\
& =\frac{1}{\pi} \int_{0}^{\infty} f_{E}(i t) K_{0}(t) \mathrm{d} x,
\end{aligned}
$$

where $f_{E}(x)=(f(x)+f(-x)) / 2$ is the even part of $f(x)$. The rest of the proof is completely analogous to the proof of theorem 5 .

Also in this case can the result be obtained by examining moments. Now,

$$
\frac{1}{2} \int_{0}^{\infty} x^{m} K_{0}(\sqrt{t}) t^{-\frac{1}{2}} \mathrm{~d} x=4^{m} \Gamma\left(\frac{1}{2}+m\right)^{2} .
$$

Figure 2 is the analogue of Figure 1 for the sine rule. The points in the left panel achieve the same asymptotic order as (twice as many) points in the right panel. 
(a)

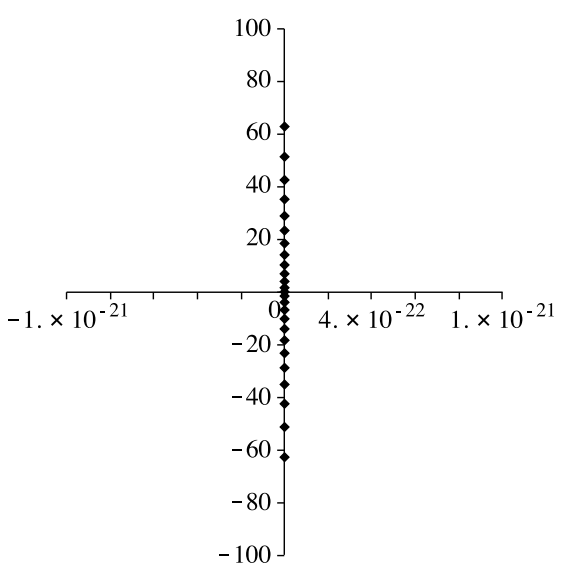

(b)

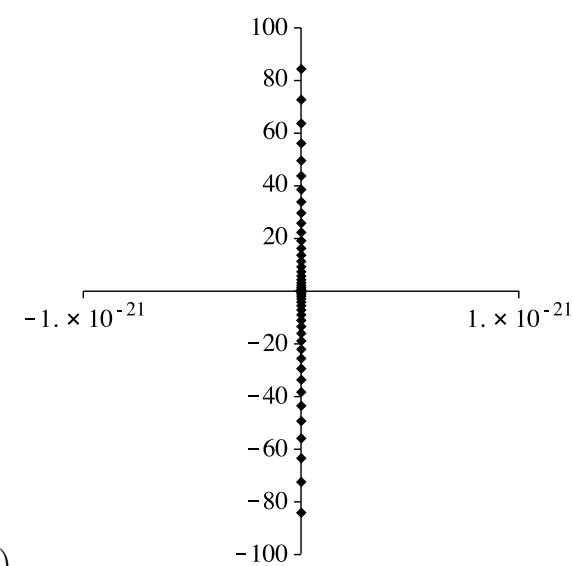

$-100$

Figure 2: (a) 25 points of the $J_{0}$-rule. (b) 25 points of each of the two rotated $K_{0}$-rule's

\subsection{Rules for $J_{\nu}$}

Moments for Bessel functions of order $\nu>0$ can be computed,

$$
\lim _{s \rightarrow 0} \int_{0}^{\infty} x^{m} J_{\nu}(x) e^{-s x} \mathrm{~d} x=2^{m} \frac{\Gamma\left(\frac{1}{2}+\frac{\nu+m}{2}\right)}{\Gamma\left(\frac{1}{2}+\frac{\nu-m}{2}\right)} .
$$

Now things are quite different from the above examples. This is indeed suggested by the fact that when rewriting in terms of $K_{\nu}$, we are in fact rewriting an entire function in terms of singular functions since

$$
K_{\nu}(z) \sim z^{-\nu}, \quad z \rightarrow 0
$$

Moreover, the singularity of $K_{\nu}$ is non-integrable for $\nu \geq 1$. The rules for the Hankel transform can however be computed using Gram-Schmidt orthogonalization. It is clear, as we see in Figure 3, that the points no longer lie on the imaginary axis, although in the approach of Wong they do.

Interestingly, it appears that the points cluster along the line $z=\nu \pi / 2$.

\subsection{An Airy rule}

In the case of Airy averaging one integrates over all reals with the Airy function $\operatorname{Ai}(x)$ as a weight. In this case extra care must be taken since only every third moment is non-zero:

$$
\lim _{s \rightarrow 0} \int_{-\infty}^{\infty} x^{m} \operatorname{Ai}(x) e^{-s x} \mathrm{~d} x= \begin{cases}3^{1-m / 3} \frac{\Gamma(m)}{\Gamma(m / 3)} & m=0,3,6, \ldots \\ 0 & \text { otherwise }\end{cases}
$$

This means that $(1, x)_{\mathrm{Ai}}=0$ and the orthogonalization process will halt. But it also means that the integral depends on only one third of the derivatives of $f$ at the origin. We can exploit this behaviour by evaluating only the part of $f$ that 


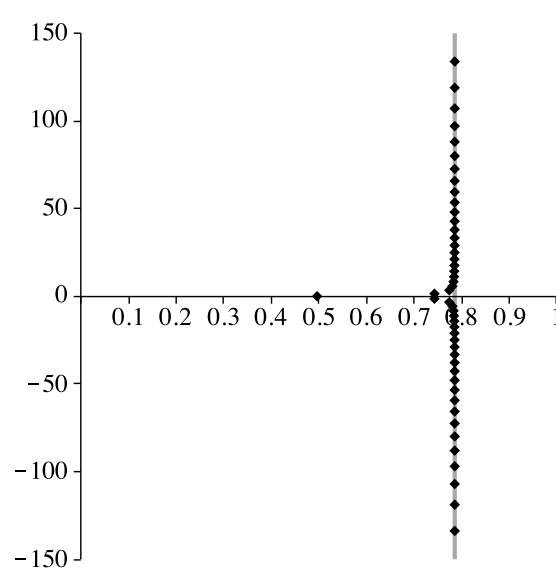

(a)

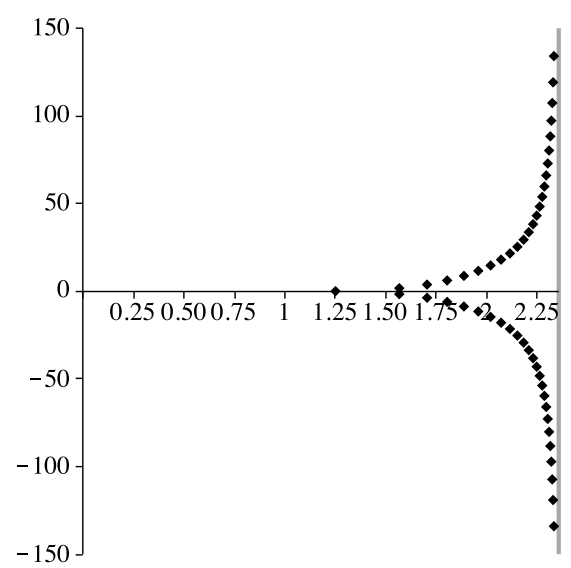

(c)

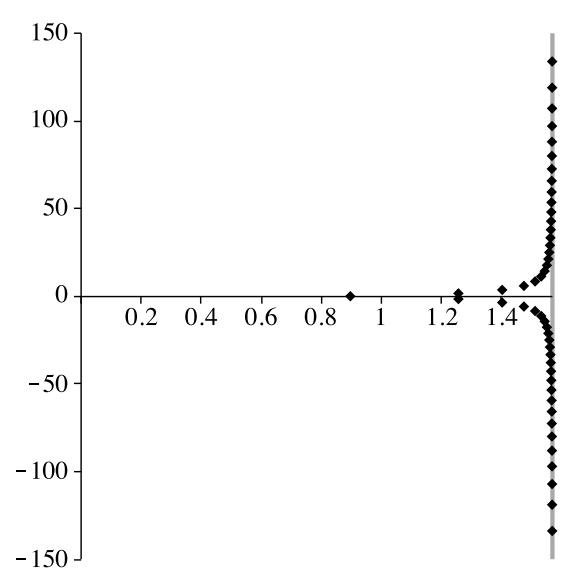

(b)

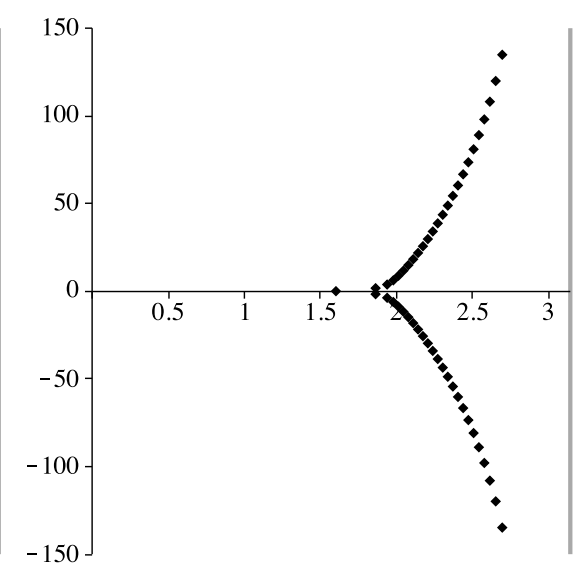

(d)

Figure 3: 50 points of (a) $J_{1 / 2}$-rule, (b) $J_{1}$-rule, (c) $J_{3 / 2}$-rule, and (d) $J_{2}$-rule. Stokes lines are indicated in grey. 
contributes to the integral. This can be achieved by taking linear combinations of evaluations of $f$ along rays in the complex plane, slightly generalizing the concept of odd and even functions, as we will now show.

Say $f$ has the Taylor series $\sum_{k} a_{k} x^{k}$. The linear combination $\frac{1}{2}[f(x)+f(-x)]$ is its even part: it has vanishing odd derivatives at the origin and the Taylor series $\sum_{k} a_{2 k} x^{2 k}$. Similarly, the linear combination $\frac{1}{3}\left[f(x)+f\left(e^{i 2 \pi / 3} x\right)+\right.$ $\left.f\left(e^{-i 2 \pi / 3} x\right)\right]$ has the Taylor series $\sum_{k} a_{3 k} x^{3 k}$. It 'selects' only every third coefficient of the Taylor series of $f$. The substitution $t=x^{3}$ subsequently makes sense and we arrive at the following result.

Theorem 7. Let $\left\{x_{j}, w_{j}\right\}_{j=1}^{n}$ be a Gaussian rule w.r.t. the weight function $\frac{1}{3} \operatorname{Ai}\left(t^{1 / 3}\right) t^{-\frac{2}{3}}$ on $[0, \infty)$. The rule $\left\{\tilde{x}_{j}, \tilde{w}_{j}\right\}_{j=1}^{3 n}$, where $\left\{\tilde{x}_{j}\right\}_{j=1}^{3 n}=\left\{x_{j}^{1 / 3}\right\} \cup\left\{x_{j}^{1 / 3} e^{2 \pi i / 3}\right\} \cup$ $\left\{x_{j}^{1 / 3} e^{-2 \pi i / 3}\right\}_{j=1}^{n}$ and $\left\{\tilde{w}_{j}\right\}_{j=1}^{3 n}=\left\{\frac{1}{3} w_{j}\right\} \cup\left\{\frac{1}{3} w_{j}\right\} \cup\left\{\frac{1}{3} w_{j}\right\}_{j=1}^{n}$, is Gaussian w.r.t. the weight $\operatorname{Ai}(x)$ on $(-\infty, \infty)$.

Proof. Use the connection formula[15, chapter 9.2],

$$
\operatorname{Ai}(z)=-e^{-2 \pi i / 3} \operatorname{Ai}\left(z e^{-2 \pi i / 3}\right)-e^{2 \pi i / 3} \operatorname{Ai}\left(z e^{2 \pi i / 3}\right) .
$$

Now write

$$
\begin{aligned}
\int_{-\infty}^{0} f(x) \operatorname{Ai}(x) \mathrm{d} x= & -e^{-2 \pi i / 3} \int_{0}^{\infty} f(-x) \operatorname{Ai}\left(-x e^{-2 \pi i / 3}\right) \mathrm{d} x \\
& -e^{2 \pi i / 3} \int_{0}^{\infty} f(-x) \operatorname{Ai}\left(-x e^{2 \pi i / 3}\right) \mathrm{d} x
\end{aligned}
$$

Assuming $f$ is analytic the integration contours can be deformed to the contour $\left(0, \infty \cdot-e^{ \pm 2 \pi i / 3}\right)$. Choosing the plus sign for the first integral and the minus sign for the other gives

$$
\int_{-\infty}^{0} f(x) \operatorname{Ai}(x) \mathrm{d} x=\int_{0}^{\infty} f\left(x e^{2 \pi i / 3}\right) \operatorname{Ai}(x) \mathrm{d} x+\int_{0}^{\infty} f\left(x e^{-2 \pi i / 3}\right) \operatorname{Ai}(x) \mathrm{d} x .
$$

From this we get the useful relation

$$
\begin{aligned}
\int_{-\infty}^{\infty} f(x) \operatorname{Ai}(x) \mathrm{d} x & =\int_{0}^{\infty}\left[f(x)+f\left(x e^{2 \pi i / 3}\right)+f\left(x e^{-2 \pi i / 3}\right)\right] \operatorname{Ai}(x) \mathrm{d} x \\
& =3 \int_{0}^{\infty} f_{3 E}(x) \operatorname{Ai}(x) \mathrm{d} x .
\end{aligned}
$$

where $f_{3 E}(x)=\left(f(x)+f\left(x e^{2 \pi i / 3}\right)+f\left(x e^{-2 \pi i / 3}\right)\right) / 3$ is a generalised even part of $f(x)$. It has an expansion where only every third term is non-zero.

Now, for the rule $\left\{x_{j}, w_{j}\right\}_{j=1}^{n}$ we have,

$$
\sum_{j=1}^{n} w_{j} x_{j}^{m}=\frac{1}{3} \int_{0}^{\infty} t^{m} \operatorname{Ai}\left(t^{1 / 3}\right) t^{-2 / 3} \mathrm{~d} t=\int_{0}^{\infty} x^{3 m} \operatorname{Ai}(x) \mathrm{d} x, \quad m=0, \ldots, 2 n-1 .
$$

This means that the rule is Gaussian for the integral (13), exactly integrating polynomials in $x^{3}$ up to order $2 n-1$. Applying the rule gives the quadrature nodes $\left\{x_{j}^{1 / 3}\right\} \cup\left\{x_{j}^{1 / 3} e^{2 \pi i / 3}\right\} \cup\left\{x_{j}^{1 / 3} e^{-2 \pi i / 3}\right\}_{j=1}^{n}$ and corresponding weights $\left\{\tilde{w}_{j}\right\}_{j=1}^{3 n}=\left\{\frac{1}{3} w_{j}\right\} \cup\left\{\frac{1}{3} w_{j}\right\} \cup\left\{\frac{1}{3} w_{j}\right\}_{j=1}^{n}$. The rule will be exact for all polynomials of degree $6 n-1$ with $3 n$ function evaluation. 
Again, the same result can be obtained by comparing moments. Now,

$$
\frac{1}{3} \int_{0}^{\infty} x^{m} \operatorname{Ai}\left(x^{1 / 3}\right) x^{-2 / 3} \mathrm{~d} x=3^{-m} \frac{\Gamma(3 m)}{\Gamma(m)} .
$$

Now note that the theorem states, since $\frac{1}{3} \operatorname{Ai}\left(x^{1 / 3}\right) x^{-2 / 3}$ is a positive function on $[0, \infty)$, that the Airy rule has nodes on the lines $t, t \cdot e^{i \pi 2 / 3}, t \cdot e^{-i \pi 2 / 3}$ and positive weights. An example of nodes for the Airy function can be seen in Figure 4.

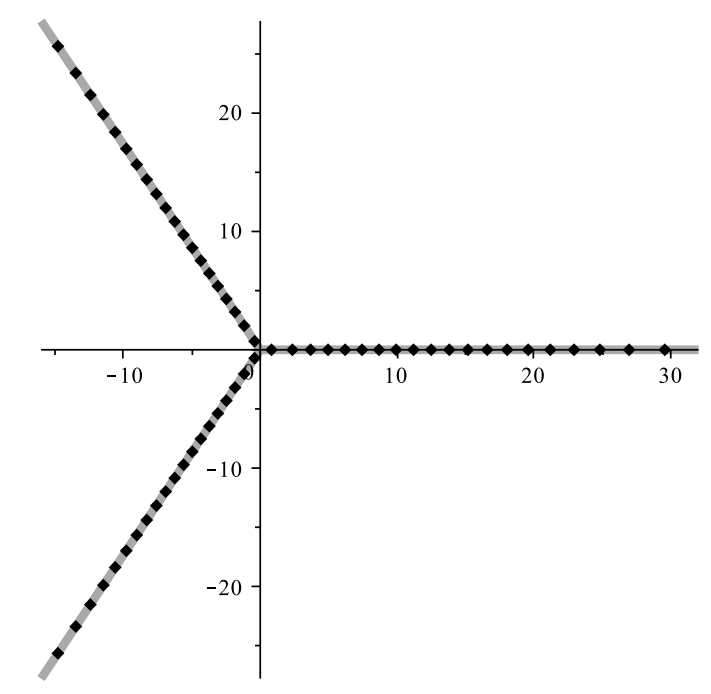

Figure 4: 60 quadrature points for $\operatorname{Ai}(x)$ over $(-\infty, \infty)$. Lines $t, t \cdot e^{i \pi 2 / 3}, t$. $e^{-i \pi 2 / 3}$ are marked in grey.

Interestingly, the rays shown in Figure 4 are precisely the Stokes lines of the Airy function.

\subsection{Rules for other kernels}

Further illustration of what the approach can yield is best demonstrated by some arbitrary examples for other kernels. We shall here see that we get quadrature nodes in non-trivial patterns.

For the integral

$$
\int_{-\infty}^{\infty} f(x) \sin \left(x^{2}\right) \mathrm{d} x
$$

moments can be computed,

$$
\lim _{s \rightarrow 0} \int_{-\infty}^{\infty} x^{m} \sin \left(x^{2}\right) e^{-s x} \mathrm{~d} x= \begin{cases}\frac{\sqrt{2}}{2} \Gamma\left(\frac{m+1}{2}\right) & m=0,2,8,10 \ldots \\ -\frac{\sqrt{2}}{2} \Gamma\left(\frac{m+1}{2}\right) & m=4,6,12,14 \ldots \\ 0 & \text { otherwise }\end{cases}
$$

Computing orthogonal polynomials is also possible, and the resulting quadrature nodes can be seen in Figure 5(a). Note that these points do not lie exactly 
on the steepest descent paths of $e^{ \pm i x^{2}}$ as one might expect. Also note that a rule for $\sin (x)$ over $(-\infty, \infty)$ can't be computed. For $\sin \left(x^{2}\right)$, the point $x=0$ is a stationary point, whereas for $\sin (x)$ the resulting integral is exponentially small (all moments evaluate to zero). For comparison, in Figure 5(b) we see the quadrature points for the kernel $\cos \left(x^{2}\right)$, for which the moments are,

$$
\lim _{s \rightarrow 0} \int_{-\infty}^{\infty} x^{m} \cos \left(x^{2}\right) e^{-s x} \mathrm{~d} x= \begin{cases}\frac{\sqrt{2}}{2} \Gamma\left(\frac{m+1}{2}\right) & m=0,2,6,8,14,16 \ldots \\ -\frac{\sqrt{2}}{2} \Gamma\left(\frac{m+1}{2}\right) & m=0,2,4,10,12 \ldots \\ 0 & \text { otherwise }\end{cases}
$$

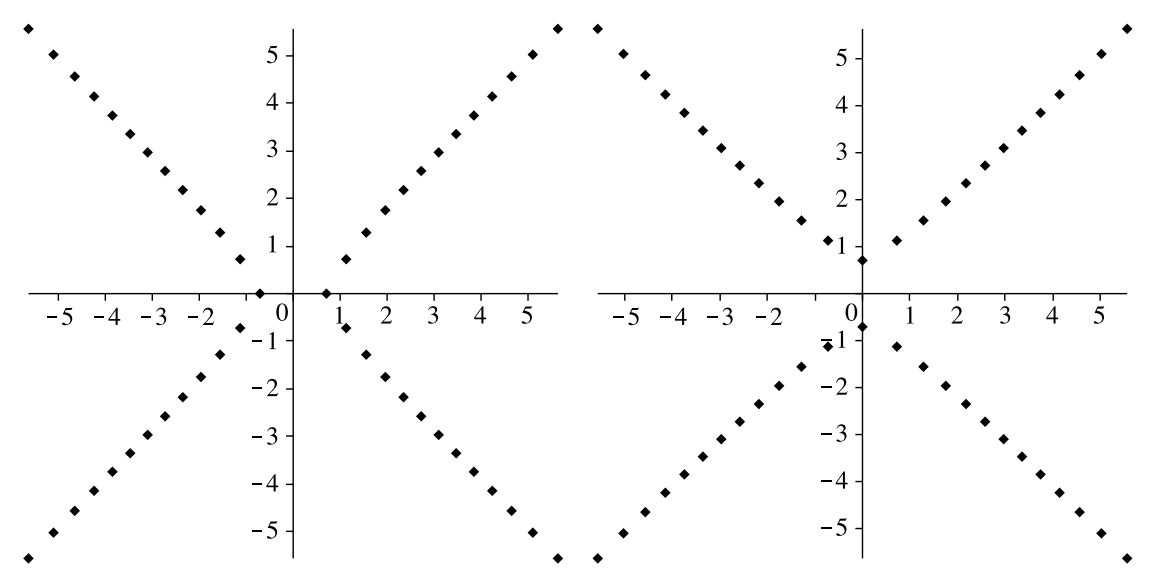

(a)

(b)

Figure 5: 50 quadrature points for the kernels $(\mathrm{a}) \sin \left(x^{2}\right)$ over $(-\infty, \infty),(\mathrm{b})$ $\cos \left(x^{2}\right)$ over $(-\infty, \infty)$

\section{Convergence of the rules}

The question of convergence of the complex Gaussian rules for integral transforms will not be discussed in great detail here, as unfortunately there is no general theory regarding the convergence of Gaussian quadrature rules on infinite intervals, even for positive weight functions. Such rules are examined on a case-by-base basis. Moreover, due to the analyticity requirements on $f$ and with the long-term aim of improving accuracy by adding points on the real line, rather than points in the complex plane, we do not advocate achieving convergence by increasing $n$. Yet, a collection of simple numerical experiments does show convergence in different cases in this section. All computations are done with 300 digits extended precision in Maple. This enables us to study the integrals for very large arguments where round off errors would swamp double precision computations. 
(a)

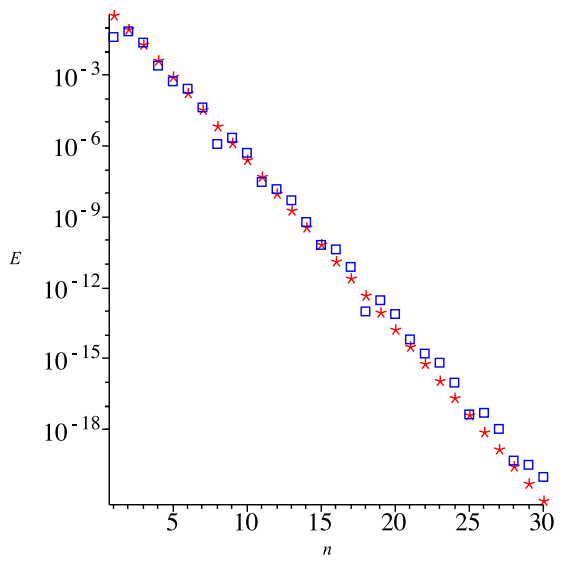

(b)

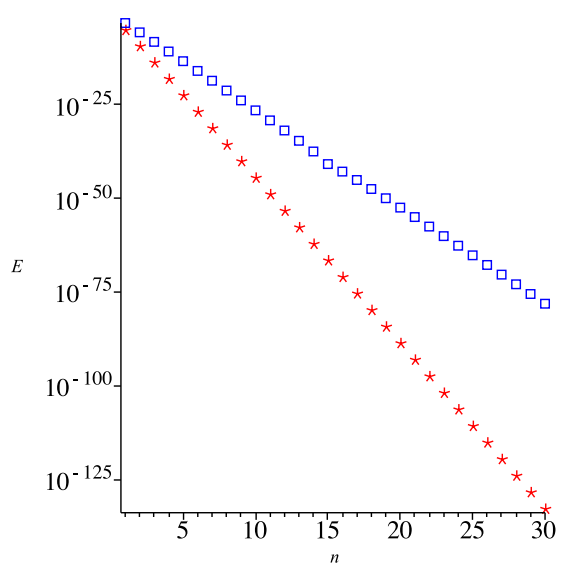

Figure 6: The error of approximating the integral (16) for $f(x)=e^{-x}$, when (a) $\omega=1$, and (b) $\omega=10$. Asterisk $(*)$ - complex Gaussian rule, square( $\square$ ) double rotated Laguerre rule

\subsection{The sine}

We wish to compare the merit of the quadrature rule with the one obtained by rewriting the sine as a combination of exponential functions, $\sin (x)=\left(e^{i x}-\right.$ $\left.e^{-i x}\right) / 2 i$, and applying rotated Gauss-Laguerre nodes. Thus, let us consider the simple test case $f(x)=e^{-x}$,

$$
I=\int_{0}^{\infty} \sin (\omega x) f(x) \mathrm{d} x=\frac{1}{2 i} \int_{0}^{\infty} f(x) e^{i \omega x} \mathrm{~d} x-\frac{1}{2 i} \int_{0}^{\infty} f(x) e^{-i \omega x} \mathrm{~d} x .
$$

We take $n$ rotated Gauss-Laguerre points for each of the two integrals on the right and compare with the same number of evaluation points when using the sine rule, i.e. $2 n$ points. For the convergence plot in figure 6 we see that the two rules appear very similar in the case where $\omega=1$ (left panel), but when increasing $\omega$ we note that the sine-rule has faster convergence (right panel). This might come as no great surprise when we take Lemma 2 into consideration.

\subsection{Evaluating Hankel transforms}

Now let us repeat the experiment with the sine function replaced by the Bessel function of order $\nu$, yielding the Hankel transform (2). Using the approach described in section $\S 2$, we first compute Gaussian rules for the weight $K_{\nu}(x)$. This shall then be compared to the rule obtained by taking $2 n$ points with the $J_{\nu}$-rule described in section $§ 3.4$. Taking the Hankel transform of order $\nu=3 / 2$ shows the same error behaviour(Figure 7). Similar behaviour is seen for other values of $\nu$. 


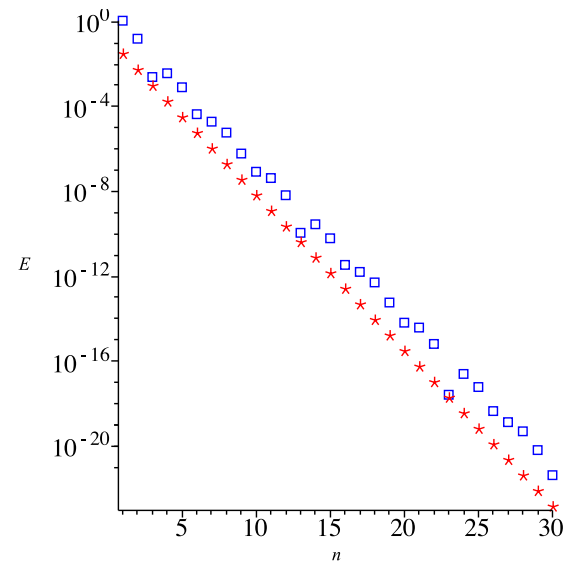

(a)

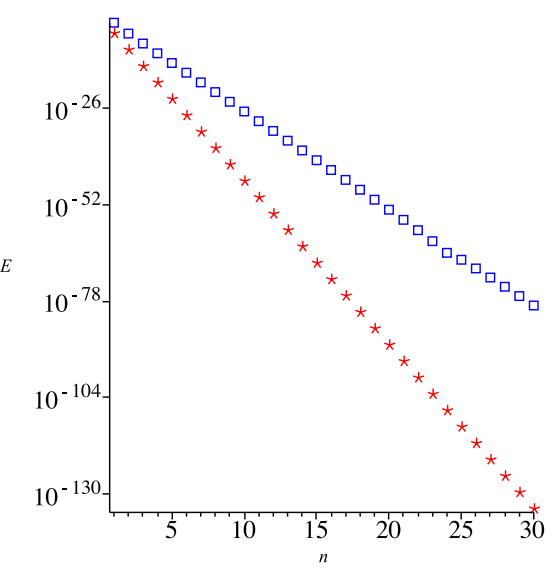

(b)

Figure 7: The error of approximating the Hankel transform of order $\nu=3 / 2$ with $f(x)=e^{-x}$ when (a) $\omega=1$, and (b) $\omega=10$. Asterisk $(*)$ - complex Gaussian rule, square( $\square)$ - double $K_{3 / 2}$-rule

\subsection{Verification of asymptotic order}

In order to verify the asymptotic order predicted by Lemma 2 we apply the $J_{3 / 2}$-rule to the integral,

$$
\int_{0}^{\infty} \frac{J_{3 / 2}(\omega x)}{(x+1)^{2}} \mathrm{~d} x,
$$

The results are tabulated in table 1 , showing in the case of $n=2$ and $n=3$ an apparent agreement with the theory. For larger $n$ the result is best shown in a plot. Figure 8 also shows a good agreement with theory for the case of equation (17).

\section{References}

[1] A. Asheim And D. Huybrechs, Asymptotic analysis of numerical steepest descent with path approximations, Foundations of Computational Mathematics, 10 (2010), pp. 647-671.

[2] N. Bleistein and R. Handelsman, A generalization of the method of steepest descent, IMA Journal of Applied Mathematics, 10 (1972), p. 211.

[3] D. Colton And R. KRess, Integral equation methods in scattering theory, vol. 271, Wiley, 1983.

[4] A. Deaño and D. Huybrechs, Complex Gaussian quadrature of oscillatory integrals, Numer. Math., 112 (2009), pp. 197-219. 


\begin{tabular}{ccc}
\multicolumn{3}{c}{$n=2$} \\
$k$ & Error & Ratio $\left(\log _{2}\right)$ \\
\hline 25 & $0.312288266242750 \mathrm{e}-5$ & \\
50 & $1.270921693016563 \mathrm{e}-7$ & 4.62 \\
100 & $4.519131062871955 \mathrm{e}-9$ & 4.81 \\
200 & $1.504642737810130 \mathrm{e}-10$ & 4.91 \\
400 & $4.851703491891264 \mathrm{e}-12$ & 4.95 \\
\multicolumn{3}{c}{$n$} \\
\hline \multicolumn{3}{c}{$n=3$} \\
50 & $7.2683352214484202 \mathrm{e}-8$ \\
100 & $8.6592757117101324 \mathrm{e}-10$ & 6.39 \\
200 & $7.0724466056311556 \mathrm{e}-12$ & 6.72 \\
400 & $5.7830301721223075 \mathrm{e}-14$ & 6.86 \\
&
\end{tabular}

Table 1: Absolute error for the $J_{3 / 2}$-rule applied to the equation (17). Ratios are apparently approaching $2^{2 n+1}$, as predicted

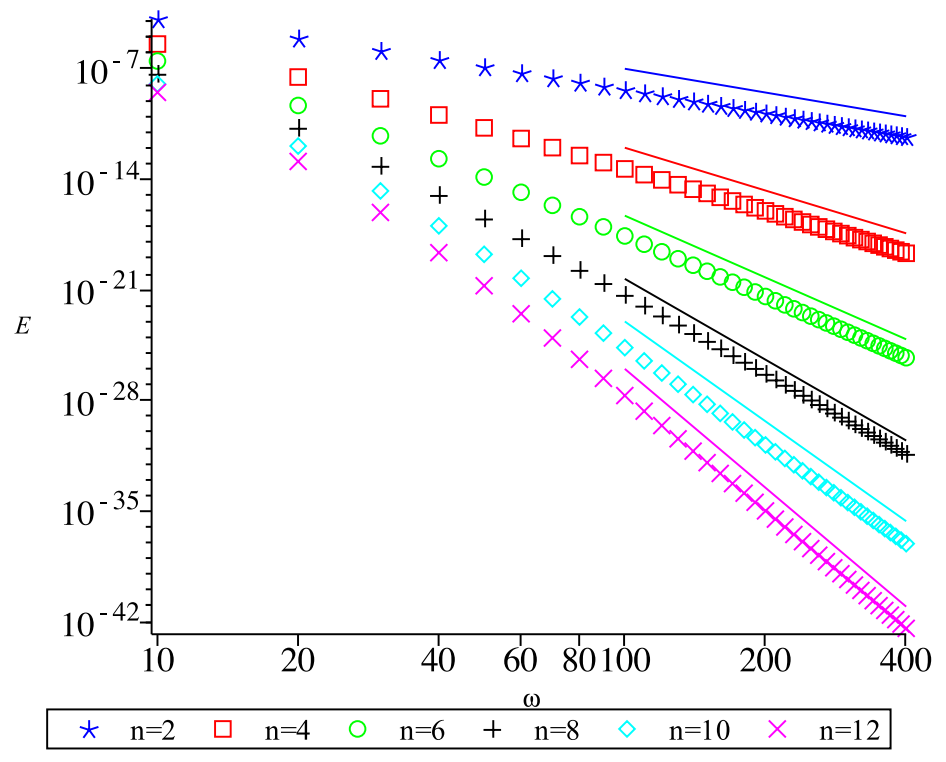

Figure 8: Absolute error of $J_{3 / 2}$-rule applied to the equation (17) as a function of $\omega$, for $n=1,2,3,4,5,6$. Lines indicate asymptotic behaviour predicted by Lemma 2 
[5] A. Deaño, D. Huybrechs, and A. Kuijlaars, Asymptotic zero distribution of complex orthogonal polynomials associated with Gaussian quadrature, Journal of Approximation Theory, 162 (2010), pp. 2202-2224.

[6] B. Englert and J. Schwinger, Statistical atom: Some quantum improvements, Physical Review A, 29 (1984), p. 2339.

[7] W. Gautschi, Computation of Bessel and Airy functions and of related Gaussian quadrature formulae, BIT Numerical Mathematics, 42 (2002), pp. $110-118$.

[8] _ Orthogonal polynomials: Computation and approximation, Oxford University Press, USA, 2004.

[9] G. H. Golub And J. H. Welsch, Calculation of Gauss quadrature rules, Mathematics of Computation, 23 (1969), pp. pp. 221-230.

[10] D. Huybrechs And S. Olver, Superinterpolation in highly oscillatory quadrature, status: published, (2010).

[11] D. Huybrechs And S. VAndewalle, On the evaluation of highly oscillatory integrals by analytic continuation, SIAM J. Numer. Anal., 44 (2006), pp. 1026-1048.

[12] —, A sparse discretisation for integral equation formulations of high frequency scattering problems, SIAM J. Sci. Comput., 29 (2007), pp. 23052328.

[13] A. IsERLES AND S. P. NøRSETt, Efficient quadrature of highly oscillatory integrals using derivatives, Proc. Roy. Soc. A., 461 (2005), pp. 1383-1399.

[14] _ Quadrature methods for multivariate highly oscillatory integrals using derivatives, Math. Comp., 75 (2006), pp. 1233-1258.

[15] F. Olver, D. Lozier, R. Boisvert, and C. Clark, NIST handbook of mathematical functions, Cambridge University Press New York, NY, USA, 2010.

[16] E. Tiтchmarsh, The Theory of Functions, Oxford University Press, USA, 1939.

[17] R. Wong, Quadrature formulas for oscillatory integral transforms, Numerische Mathematik, 39 (1982), pp. 351-360. 